\title{
Predictors of Visual Recovery in Patients with Macular Edema Secondary to Central Retinal Occlusion After Treatment with Conbercept
}

\section{Qi Zhang}

Yijishan Hospital of Wannan Medical College

\section{Yinfen Hou}

Yijishan Hospital of Wannan Medical College

\section{Xiao Cao}

Yijishan Hospital of Wannan Medical College

\section{Rongrong Zhang}

Yijishan Hospital of Wannan Medical College

\section{Yinping Liu}

Yijishan Hospital of Wannan Medical College

\section{Chenghua Wei}

Yijishan Hospital of Wannan Medical College

\section{Changfan Wu}

Yijishan Hospital of Wannan Medical College

\section{Lixin Mei}

Yijishan Hospital of Wannan Medical College

Pengfei Zhang ( $\nabla$ zhangpengfei1023@126.com )

Yijishan Hospital of Wannan Medical College

\section{Research Article}

Keywords: CRVO-ME, conbercept, best corrected visual acuity, predictive factor

Posted Date: June 2nd, 2021

DOI: https://doi.org/10.21203/rs.3.rs-540790/v1

License: (c) (1) This work is licensed under a Creative Commons Attribution 4.0 International License. Read Full License 


\section{Abstract \\ Background}

The use of Spectral domain optical coherence tomography (SD-OCT) to evaluate the predictors of visual acuity-recovery in patients treated with conbercept for macular edema (ME) secondary to central retinal vein occlusion (CRVO) has rarely been seen.We collected 24 CRVO-ME patients with different OCT measures at 6 months follow-up to identify the factors that are most strongly correlated with the bestcorrected visual acuity (BCVA) post-treatment in CRVO-ME patients treated with conbercept

\section{Purpose}

To evaluate the effectiveness of intravitreal conbercept injections for the treatment of CRVO-ME and to determine the major predictors of best-corrected visual acuity (BCVA) post-treatment.

\section{Methods}

A retrospective study methodology was used. Twenty-four eyes from 24 patients with CRVO-ME were enrolled in the study. After an initial intravitreal injection of conbercept $(0.5 \mathrm{mg} / 0.05 \mathrm{ml})$, monthly injections for up to 6 months were given following a $1+$ PRN (pro re nata) regimen. Data collected at monthly intervals included measurements of the logMAR BCVA, central subfield thickness (CST), macular volume (MV), photoreceptor layer thickness (PLT), outer nuclear layer thickness (ONLT), and the disrupted ellipsoid zone (DEZ). The correlation between BCVA, before and after injections, and each of CST, MV, PLT, ONLT, DEZ was analyzed.

\section{Results}

The logMAR BCVA in months 3 and 6 post-injection was significantly improved relative to the baseline. In this same period the CST, MV, PLT, ONLT and DEZ were also significantly improved relative to the baseline. There was a negative correlation between PLT and logMAR BCVA at months 3 and $6(r=-0.549$, $P<0.001 ; r=-0.087, P<0.001)$.

\section{Conclusion}

Intravitreal injection of conbercept is an effective treatment for CRVO-ME. With six months of follow-up, logMAR BCVA and CST, MV, PLT, ONLT, DEZ improved. PLT was negatively correlated with the visual function in CRVO-ME patients after conbercept treatment, which may be a predictor of vision recovery in patients with CRVO-ME. 


\section{Background}

Central retinal vein occlusion (CRVO) is one of the leading causes of vision loss among people aged 50 years or older. The incidence rate of CRVO in this group is approximately 1.36\%.[1] Macular edema (ME) is the most common complication and a major cause of visual impairment in people with CRVO.[2]

During CRVO formation, vascular endothelial growth factor (VEGF) levels increase resulting in an increase in the permeability of the retinal vessel walls. This causes the breakdown of the blood retinal barrier and ultimately leads to ME.[3] Many studies have shown that intravitreal anti-VEGF therapy is considered safe and effective for the treatment of CRVO-ME.[4, 5] Conbercept, an anti-VEGF medication, is a humanized, soluble, VEGF receptor (VEGFR) protein comprising extracellular domain-2 of VEGFR1, and extracellular domains-3 and-4 of VEGFR-2. Intravitreal injection of conbercept has been proven safe and effective for the treatment of CRVO-ME.[6, 7] However, clinically, repeated administration and follow-up visits are required, and some patients do not respond to treatment or have a poor visual outcome after repeated administration. $[8,9]$ Thus, it is very important to explore the predictive factors of a successful clinical outcome from conbercept therapy.

SD-OCT has been demonstrated to be an effective technique for analyzing macular edema through the evaluation of retinal structures. For example, using this technique, $\mathrm{Xu}$ et al. discovered that the ganglion cell layer and the inner plexiform layer are associated with visual gains in patients with diabetic macular edema.[10] Liu et al. discovered that external limiting membrane integrity is a prediction factor of visual function in CRVO-ME patients that have received ranibizumab injections.[11] However, SD-OCT assessment is still rarely to predict the visual acuity in CRVO-ME patients after conbercept therapy.

In this study, we investigated the retinal microstructure in patients with CRVO-ME after treatment with intravitreal conbercept during six-months follow-up period. We aim to identify the factors that are most strongly correlated with the best-corrected visual acuity (BCVA) post-treatment.

\section{Materials And Methods}

\section{Patients and Inclusion criteria.}

This was a retrospective study. The patient group in this study consisted of 24 individuals diagnosed with CRVO-ME (24 eyes). All patients were examined and treated in the Department of Ophthalmology, the First Affiliated Hospital of Wannan Medical College, China, from May 2018 to February 2019. Inclusion criteria were: (1) fundus fluorescein angiography diagnosis of CRVO; (2) OCT showed macular edema with macular thickness > $250 \mu \mathrm{m}$; (3) no prior treatment for CRVO-ME; (4) intraocular pressure within the normal range; (5) no other eye diseases present, including severe cataract, glaucoma, uveitis, fundus diseases, myopia more than 3.00 diopters (D); (6) no history of eye surgery; and (7) no other serious medical diseases. All patients had a detailed understanding of the treatment benefits and risks, and of the study's medical ethics requirements. The study conformed to the tenets of the Declaration of Helsinki and was approved by the Ethical Review Committee of Yijishan Hospital of Wannan Medical College, 
China (LLSC-2021-095). Written informed consent was obtained from each patient prior to participation in the study.

\section{Treatments and Ophthalmic examination.}

All patients initially received one intravitreal injection of $0.5 \mathrm{mg} / 0.05 \mathrm{~mL}$ of Conbercept (Chengdu Kang Hong Biotech Co, Ltd, Sichuan, China) and PRN (pro re nata) thereafter. All patients were evaluated monthly, including fluorescein angiography (FA) and optical coherent tomography (OCT) (Heidelberg Engineering, Heidelberg, Germany). If persistent or recurrent edema was detected, the $1+P R N$ regimen was adopted.

For all participants, we used OCT for data collection, including the logMAR BCVA, central subfield thickness (CST), macular volume (MV), photoreceptor layer thickness (PLT), outer nuclear layer thickness (ONLT), and disrupted ellipsoid zone (DEZ). Before the examination, the patients may be given Compound Topicamide eye drops for convenience of examination. During the examination, the examiner aims the lens at the patient's eye sitting directly in front of OCT, and lets the patient look at a fixed point of view and adjust the fixed point of view until a clear fundus image and OCT scanning line are presented on the fundus imaging display. At this time, relevant data required by OCT can be displayed and collected three times and took the average of them automatically according to the OCT instrument.

\section{Outcome Measurements.}

All patients were followed for 6 months post-treatment. The logMAR BCVA, central subfield thickness (CST), macular volume (MV), photoreceptor layer thickness (PLT), outer nuclear layer thickness (ONLT), and disrupted ellipsoid zone (DEZ) were evaluated at the baseline, week 1, and at months 1,3 , and 6 posttreatment.

\section{Statistical Analyses.}

All analyzes were conducted using SPSSv.18.0 for Windows (SPSS, Chicago, IL). Quantitative data that were normally distributed were analyzed using ANOVA. The correlation assesses between BCVA after medication and the OCT parameters using Pearson's analysis. All statistical tests were two-sided. A $P$ value of $<0.05$ was considered statistically significant.

\section{Results}

\section{Demographic and clinical characteristics.}

Twenty-six eyes of 26 patients (11 females, 15 males) with CRVO-ME met the inclusion criteria between February 2011 and May 2016 (Table 1). The mean age of the participants was $57.70 \pm 12.91$ years. The mean intraocular pressure (IOP) of patients was $12.64 \pm 2.13 \mathrm{mmHg}$. All patients received the $1+P R N$ regimen. The total number of injections was 53 , while the average number of injections per patient was $2.03 \pm 0.92$ (minimum =1; maximum $=4$ ). Three cases of subconjunctival hemorrhage occurred post- 
injection, with each undergoing self-absorption after 1 week of observation. There were no special complications in the remaining cases.

Table 1

Baseline characteristics of CRVO-ME patients

\begin{tabular}{|ll|}
\hline & CRVO-ME group \\
\hline Numble & 26 \\
\hline Age (years) & $57.70 \pm 12.91$ \\
\hline Sex $(\mathrm{F} / \mathrm{M})$ & $11 / 15$ \\
\hline BCVA $(\mathrm{logMAR})$ & $1.00 \pm 0.73$ \\
\hline IOP $(\mathrm{mmHg})$ & $12.64 \pm 2.13$ \\
\hline Mean CST $(\mu \mathrm{m})$ & $747.58 \pm 149.38$ \\
\hline Mean MV $\left(\mu \mathrm{m}^{3}\right)$ & $747.58 \pm 149.38$ \\
\hline Mean PLT $(\mu \mathrm{m})$ & $58.96 \pm 8.56$ \\
\hline Mean ONLT $(\mu \mathrm{m})$ & $432.58 \pm 45.24$ \\
\hline Mean DEZ $(\mu \mathrm{m})$ & $2921.04 \pm 536.37$ \\
\hline
\end{tabular}

\section{Baseline and follow-up Characteristics.}

The mean logMAR BCVA was $1.00 \pm 0.73$ at baseline, and the respective values for the mean logMAR BCVA in week 1 , and months 1,3 , and 6 were $0.53 \pm 0.33,0.44 \pm 0.29,0.40 \pm 0.25$, and $0.36 \pm 0.08$. Comparison of the logMAR BCVA pre-injection and post-injection showed a statistically significant difference in months $3(P=0.007)$ and $6(P=0.003)$ post-injection (Fig. 1A).

The mean CST was $747.58 \pm 149.38 \mu \mathrm{m}$ at baseline, and the respective values for the mean CST in week 1 , and in months 1,3 , and 6 were $609.92 \pm 187.73 \mu \mathrm{m}, 404.50 \pm 129.49 \mu \mathrm{m}, 299.50 \pm 74.24 \mu \mathrm{m}$, and 274.08 $\pm 29.30 \mu \mathrm{m}$. Mean CST decreased between the baseline period and week $1(P=0.003)$, month $1(p \leq$ $0.001)$, month 3 ( $p \leq 0.001)$, and month 6 ( $p \leq 0.001)$ post-injection. Mean CST decreased between week 1 post-injection and month $1(p \leq 0.001)$, month $3(p \leq 0.001)$, and month $6(p \leq 0.001)$ post-injection. Mean CST also decreased between month 1 post-injection and both month $3(P=0.018)$ and month $6(P$ $=0.004$ ) post-injection (Fig. 1B).

The mean MV was $30.87 \pm 2.63 \mu \mathrm{m}^{3}$ at baseline, and the respective values for the mean $\mathrm{MV}$ in week 1 , and in months 1,3 , and 6 were $28.62 \pm 1.41 \mu \mathrm{m}^{3}, 12.70 \pm 2.10 \mu \mathrm{m}^{3}, 11.57 \pm 1.35 \mu \mathrm{m}^{3}$, and $10.93 \pm 0.52 \mu \mathrm{m}^{3}$. Mean MV in decreased between the baseline and week $1(P=0.005)$, month $1(p \leq 0.001), 3(p \leq 0.001)$, and $6(p \leq 0.001)$ post-injection. Mean MV decreased between week 1 post-injection and month $1(p \leq$ 
0.001), month 3 ( $p \leq 0.001)$, and month $6(p \leq 0.001)$ post-injection. Mean MV also decreased between month 1 post-injection and month 6 post-injection $(P=0.023$, Fig. 1C).

The mean PLT was $58.96 \pm 8.56 \mu \mathrm{m}$ at baseline, and the respective values for the mean MV in week 1 , and in months 1,3 , and 6 were $51.85 \pm 8.22 \mu \mathrm{m}, 39.04 \pm 9.03 \mu \mathrm{m}, 20.92 \pm 5.36 \mu \mathrm{m}$, and $19.15 \pm 1.68 \mu \mathrm{m}$. PLT decreased between the baseline period and week $1(P=0.006)$, month $1(p \leq 0.001)$, month $3(P=0.006)$, and month $6(P=0.006)$ post-injection. PLT decreased between week 1 post-injection and month 1 ( $p \leq$ $0.001)$, month 3 ( $p \leq 0.001)$, and month 6 ( $p \leq 0.001)$ post-injection. PLT also decreased between month 1 post-injection and both month $3(p \leq 0.001)$ and month $6(p \leq 0.001)$ post-injection (Fig. 1D).

The mean ONLT was $432.58 \pm 45.24 \mu \mathrm{m}$ at baseline, and the respective values for the mean MV in week 1 , and in months 1,3 , and 6 were $389.31 \pm 42.45 \mu \mathrm{m}, 317.96 \pm 41.57 \mu \mathrm{m}, 217.04 \pm 48.86 \mu \mathrm{m}$, and $157.92 \pm$ $8.87 \mu \mathrm{m}$. The ONLT decreased between the baseline period and week $1(P=0.002)$, month $1(P \leq 0.001)$, month $3(P \leq 0.001)$, and month $6(p \leq 0.001)$ post-injection. The ONLT decreased between week 1 postinjection and month $1(P \leq 0.001)$, month $3(P \leq 0.001)$, and month $6(P \leq 0.001)$ post-injection. The ONL also decreased between month 1 post-injection and both month $3(P \leq 0.001)$ and month $6(P \leq 0.001)$ post-injection. Furthermore, the ONLT decreased between month 3 and month 6 post-injection $(P=0.001$, Fig. 1E).

The mean DEZ is $2921.04 \pm 536.37 \mu \mathrm{m}$ at baseline, and the respective values for the mean MV in week 1 , and in months 1,3 , and 6 were $2401.23 \pm 543.33 \mu \mathrm{m}, 1944.73 \pm 522.30 \mu \mathrm{m}, 1440.88 \pm 542.88 \mu \mathrm{m}$, and $1245.42 \pm 105.19 \mu \mathrm{m}$. The DEZ decreased between the baseline and week $1(P=0.002)$, month $1(P \leq$ 0.001), month $3(P \leq 0.001)$, and month $6(P \leq 0.001)$ post-injection. The DEZ decreased between week 1 post-injection and month $1(P=0.005)$, month $3(P \leq 0.001)$, and month $6(P \leq 0.001)$ post-injection. The DEZ also decreased between month 1 post-injection and both month $3(P=0.002)$ and month $6(P \leq$ 0.001) post-injection (Fig. 1F).

\section{Correlation Analysis of BCVA after injection.}

The results of the Pearson's correlation analysis showed that there was a statistically significant correlation between the baseline of PLT and the post-injection BCVA correlated at month $3(r=-0.549, P<$ $0.001)$ and month $6(r=-0.087, P<0.001$, Fig. 2$)$.

\section{Discussion}

Macular edema is the major complication secondary to central retinal vein occlusion that can lead to severe impairment of central vision.[12] Previous studies have shown that CRVO-ME can destroy the normal structure of macular causing photoreceptor dysfunction.[13,14] Therefore, it is necessary to assess the changes in macular structure and visual function associated with gains in visual acuity after an intravitreal conbercept injection. In this study, we found that after conbercept injection CST, MV, PLT, $\mathrm{ONL}$, and DEZ all improved with observation time relative to baseline measurements. The mean logMAR BCVA improved significantly in the 3 months after treatment and stabilized by 6 months. Furthermore, we 
showed that the baseline of PLT and BCVA at months 3 and 6 have a negative correlation, which indicated that PLT may be a predictor for vision recovery of CRVO-ME.

Although treatment of macular edema typically follows a $3+P R N$ regimen due to regional economic influences,[15] our treatment regimen was monthly intravitreal conbercept injections for 1 month followed by $1+$ PRN regimen. It was found that the structure and function of the macular area were improved relative to the baseline and the visual acuity improved over the duration of the study. This suggests that the treatment program is effective for treating CRVO-ME. The results reported here are consistent with previous studies.[16, 17]

Previous studies have noted that vision recovery was limited even though retinal thickness was reduced to normal levels after treatment.[18, 19] The reason for this is not clear. It is known that macular edema was noted mostly in the outer nuclear layer, causing photoreceptor cell loss and fovea dysfunction.[19] Akagi-Kurashige et al. found that macular photoreceptor abnormalities of RVO patients could cause a decrease in parafoveal cone density and disrupt the cone mosaic spatial arrangement.[20] Therefore, the assessment of the retinal layers, especially those consisting of photoreceptor layers, is useful for evaluating visual prognosis.

In our study, we found a negative correlation between BCVA and PLT. The photoreceptor layer is the area where photoreceptor cells are distributed on the OCT. Therefore, the variation of photoreceptor thickness reflects the loss of photoreceptors. In this study, it was found that PLT is one of the most important factors in predicting the ultimate outcome of vision when we treat CRV-ME patients using conbercep.

There are some methodological limitations that should be considered when evaluating the results of this study. First, the study was performed at a single center and the sample size was relatively small. Second, CRVO is a chronic disease, while the observation time in this study is short. It is therefore difficult to fully assess the long-term prognosis with conbercept treatment. Third, we manually measured the various retinal layer thicknesses three times and took the average of them, which increases the risk of any potential observer bias affecting the results. The use of automated software would have allowed a more objective measurements to be made. A larger sample size and longer follow-up period will be needed to validate our results.

In sum, the study demonstrated the efficacy of conbercept treatment for CRVO-ME using a $1+$ PRN regimen. Improvements with observation time over the 6 months follow-up period were found in the central subfield thickness, macular volume, photoreceptor layer thickness, outer nuclear layer and disrupted ellipsoid zone. The mean logMAR BCVA are improved significantly in the 3 months after treatment and stabilized by 6 months post-treatment. PLT is associated with the visual function in CRVOME patients after conbercept treatment and may be a predictor for vision recovery.

\section{Abbreviations}

SD-OCT 
Spectral domain optical coherence tomography; BCVA:Best-corrected visual acuity; CRVO-ME:macular edema secondary to central retinal vein occlusion; CST:central subfield thickness; MV:macular volume; PLT:photoreceptor layer thickness; ONLT:outer nuclear layer thickness; DEZ:the disrupted ellipsoid zone

\section{Declarations}

\section{Acknowledgements}

Not applicable.

\section{Authors' contributions}

QZ, PFZ conceived and designed the study. QZ, YFH, XC, RRZ, YPL, CHW, CFW, LXM participated in information gathering and editing, analyzed and interpreted all the data. QZ wrote the first draft of manuscript. Other authors reviewed and edited the manuscript. QZ reviewed and approved the final the version published. All authors read and approved the final manuscript.

\section{Foundation}

This study was funded by the National Science Foundation of Anhui Province, China (grant No. 1808085MH253) and the Training Program for Talents from Higher Education of Anhui Province, China (grant No. gxgwfx2019034). The funders had no role in study design, data collection and analysis, decision to publish or preparation of the manuscript. None of authors have any financial interest in any of the material described herein.

\section{Availability of data and materials}

The data are available from the corresponding author upon reasonable request.

\section{Ethics approval and consent to participate}

This study was approved by the Ethics Committee of Wuhu Yijishan Hospital of Wannan Medical College, China (LLSC-2021-095). Written informed consent was obtained from each patient prior to participation in the study.

\section{Consent for publication}

We have obtained explicit written informed consent to publish all data (including individual details, images or videos) related to the study

\section{Competing interests}

The authors declare that they have no competing interests.

\section{Author details}




\section{References}

1. Ehlers JP, Fekrat S. Retinal vein occlusion: beyond the acute event. Surv Ophthalmol. 2011; 56(4):281-299.

2. Rhoades W, Dickson D, Nguyen QD, Do DVJTJoO. Management of macular edema due to central retinal vein occlusion - The role of aflibercept. 2017; 7(2):70-76.

3. McIntosh RL, Rogers SL, Lim L, Cheung N, Wang JJ, Mitchell P, Kowalski JW, Nguyen HP, Wong TY. Natural history of central retinal vein occlusion: an evidence-based systematic review. Ophthalmology. 2010; 117(6):1113-1123 e1115.

4. Daien V, Eldem BM, Talks JS, Korobelnik JF, Mitchell P, Finger RP, Sakamoto T, Wong TY, Evuarherhe $\mathrm{O}$, Carter $\mathrm{G}$ et al. Real-world data in retinal diseases treated with anti-vascular endothelial growth factor (anti-VEGF) therapy - a systematic approach to identify and characterize data sources. BMC Ophthalmol. 2019; 19(1):206.

5. Callizo J, Ziemssen F, Bertelmann T, Feltgen N, Vogeler J, Koch M, Eter N, Liakopoulos S, SchmitzValckenberg S, Spital G. Real-World Data: Ranibizumab Treatment For Retinal Vein Occlusion In The OCEAN Study. Clin Ophthalmol. 2019; 13:2167-2179.

6. Zhang J, Liang Y, Xie J, Li D, Hu Q, Li X, Zheng W, He R. Conbercept for patients with age-related macular degeneration: a systematic review. BMC Ophthalmol. 2018; 18(1):142.

7. Deng Y, Zhong QW, Zhang AQ, Cai XJ, Lu MZ, Zhang SC, Su LS, Chen H, Lin Y, Sun LM et al. Microvascular changes after conbercept therapy in central retinal vein occlusion analyzed by optical coherence tomography angiography. International journal of ophthalmology. 2019; 12(5):802-808.

8. Ip MS, Oden NL, Scott IU, VanVeldhuisen PC, Blodi BA, Ghuman T, Baker CW. Month 12 Outcomes After Treatment Change at Month 6 Among Poor Responders to Aflibercept or Bevacizumab in Eyes With Macular Edema Secondary to Central or Hemiretinal Vein Occlusion: A Secondary Analysis of the SCORE2 Study. JAMA ophthalmology. 2019; 137(3):281-287.

9. Eldeeb M, Chan EW, Dedhia CJ, Mansour A, Chhablani J. One-year outcomes of ziv-aflibercept for macular edema in central retinal vein occlusion. American journal of ophthalmology case reports. 2017; 8:58-61.

10. Xu Y, Qu Y, Suo Y, Gao J, Chen X. Correlation of retinal layer changes with vision gain in diabetic macular edema during conbercept treatment. 2019; 19(1):123.

11. Liu H, Li S, Zhang Z, Shen J. Predicting the visual acuity for retinal vein occlusion after ranibizumab therapy with an original ranking for macular microstructure. Exp Ther Med. 2018; 15(1):890-896.

12. Huang P, Niu W, Ni Z, Wang R, Sun X. A meta-analysis of anti-vascular endothelial growth factor remedy for macular edema secondary to central retinal vein occlusion. PLoS One. 2013; 8(12):e82454. 
13. Shin HJ, Chung H, Kim HC. Association between integrity of foveal photoreceptor layer and visual outcome in retinal vein occlusion. Acta ophthalmologica. 2011; 89(1):e35-40.

14. Altunel O, Duru N, Goktas A, Ozkose A, Goktas E, Atas M. Evaluation of foveal photoreceptor layer in eyes with macular edema associated with branch retinal vein occlusion after ozurdex treatment. International ophthalmology. 2017; 37(2):333-339.

15. Luo W, Jia F, Liu M, Wang Y, Zhang T. The Analysis of Correlative Factors of Visual Acuity with Intravitreal Conbercept Injection in Macular Edema Associated with Branch Retinal Vein Occlusion. Journal of ophthalmology. 2018; 2018:7348153.

16. Osaka R, Muraoka Y, Miwa Y, Manabe K, Kobayashi M, Takasago Y, Ooto S, Murakami T, Suzuma K, lida $Y$ et al. Anti-Vascular Endothelial Growth Factor Therapy for Macular Edema following Central Retinal Vein Occlusion: 1 Initial Injection versus 3 Monthly Injections. Ophthalmologica Journal international d'ophtalmologie International journal of ophthalmology Zeitschrift fur Augenheilkunde. 2018; 239(1):27-35.

17. Miwa Y, Muraoka Y, Osaka R, Ooto S, Murakami T, Suzuma K, Takahashi A, lida Y, Yoshimura N, Tsujikawa A. RANIBIZUMAB FOR MACULAR EDEMA AFTER BRANCH RETINAL VEIN OCCLUSION: One Initial Injection Versus Three Monthly Injections. Retina (Philadelphia, Pa). 2017; 37(4):702-709.

18. Al-Zamil WM, Yassin SA. Recent developments in age-related macular degeneration: a review. Clin Interv Aging. 2017; 12:1313-1330.

19. Yu JJ, Thomas AS, Berry D, Yoon S, Fekrat S, Grewal DS. Association of Retinal Inner Layer Disorganization With Ultra-Widefield Fluorescein Angiographic Features and Visual Acuity in Branch Retinal Vein Occlusion. Ophthalmic surgery, lasers \& imaging retina. 2019; 50(6):354-364.

20. Akagi-Kurashige Y, Tsujikawa A, Ooto S, Makiyama Y, Muraoka Y, Kumagai K, Uji A, Arichika S, Murakami T, Miyamoto $\mathrm{K}$ et al. Retinal microstructural changes in eyes with resolved branch retinal vein occlusion: an adaptive optics scanning laser ophthalmoscopy study. American journal of ophthalmology. 2014; 157(6):1239-1249.e1233.

\section{Figures}




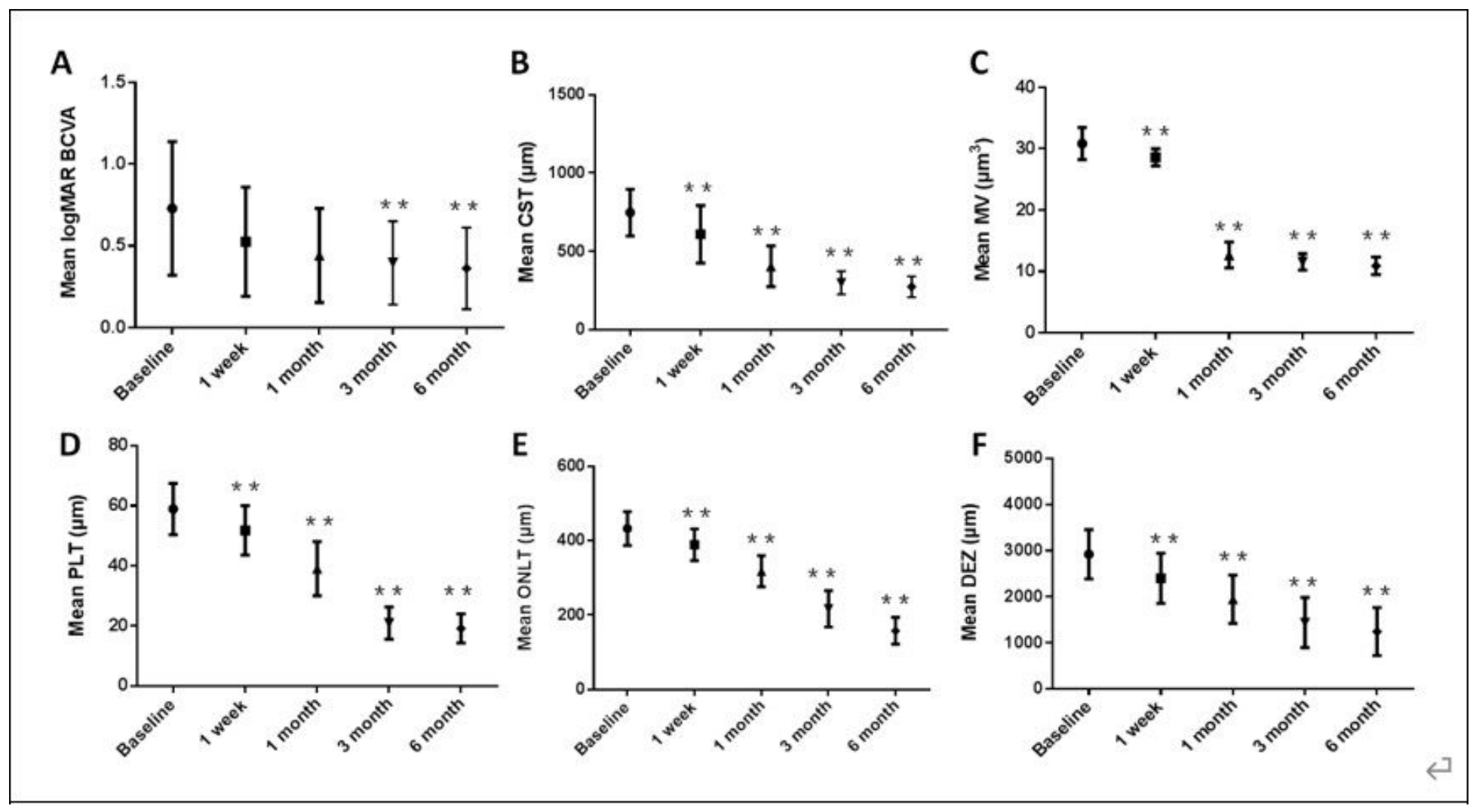

\section{Figure 1}

Comparison of BCVA (A), CST (B), MV (C), PLT (D), and ONLT (E) and DEZ (F) from baseline to month 6. The data are expressed as the mean $\pm S D$, Compared with baseline ( $\mathbb{Q} \mathrm{p}<0.001)$. LogMAR BCVA of month $3(P=0.007)$ and month $6(P=0.003)$ post-injection significantly decreased compared to baseline (A). Compared to baseline, CST decreased at week $1(P=0.003)$, month $1(P \leq 0.001)$, month $3(P \leq$ 0.001), and month $6(P \leq 0.001)$ post-injection. Compared to week 1 post-injection, CST decreased at month 1 ( $P \leq 0.001)$, month $3(P \leq 0.001)$, and month 6 ( $P \leq 0.001)$ post-injection. Compared to month 1 post-injection, CST decreased at month $3(P=0.018)$ and month $6(P=0.004)$ post-injection $(B)$.

Compared to baseline, MV significantly decreased at week $1(P=0.005)$, month $1(P \leq 0.001)$, month 3 ( $P$ $\leq 0.001)$, and month $6(\mathrm{P} \leq 0.001)$ post-injection. Compared to week 1 post-injection, MV decreased at month $1(P \leq 0.001)$, month $3(P \leq 0.001)$, and month $6(P \leq 0.001)$ post-injection. Mean MV also decreased between month 1 post-injection and month 6 post-injection $(P=0.023$, Fig $C)$. Compared to baseline, PLT decreased at week $1(P=0.006)$, month $1(P \leq 0.001)$, month $3(P=0.006)$, and month 6 ( $P$ $=0.006)$ post-injection. Compared to week 1 post-injection, PLT decreased at month $1(P \leq 0.001)$, month $3(\mathrm{P} \leq 0.001)$, and month $6(\mathrm{P} \leq 0.001)$ post-injection. Compared to month 1 post-injection, $\mathrm{PLT}$ decreased at month $3(P \leq 0.001)$ and month $6(P \leq 0.001)$ post-injection (Fig $D)$. Compared to baseline, The ONLT decreased at week $1(P=0.002)$, month $1(P \leq 0.001)$, month $3(P \leq 0.001)$, and month $6(P \leq$ 0.001) post-injection. Compared to week 1 post-injection, ONLT decreased at month 1 ( $P \leq 0.001)$, month $3(P \leq 0.001)$, and month $6(P \leq 0.001)$ post-injection. The ONL also decreased between month 1 postinjection and both month $3(P \leq 0.001)$ and month $6(P \leq 0.001)$ post-injection. Furthermore, the ONLT decreased between month 3 and month 6 post-injection $(P=0.001$, Fig $E)$. Compared to baseline, DEZ 
decreased at week $1(P=0.002)$, month $1(P \leq 0.001)$, month $3(P \leq 0.001)$, and month $6(P \leq 0.001)$ post-injection. Compared to week 1 post-injection, DEZ decreased at month $1(P=0.005)$, month $3(P \leq$ 0.001), and month $6(P \leq 0.001)$ post-injection. The $D E Z$ also decreased between month 1 post-injection and both month $3(P=0.002)$ and month $6(P \leq 0.001)$ post-injection (Fig F).

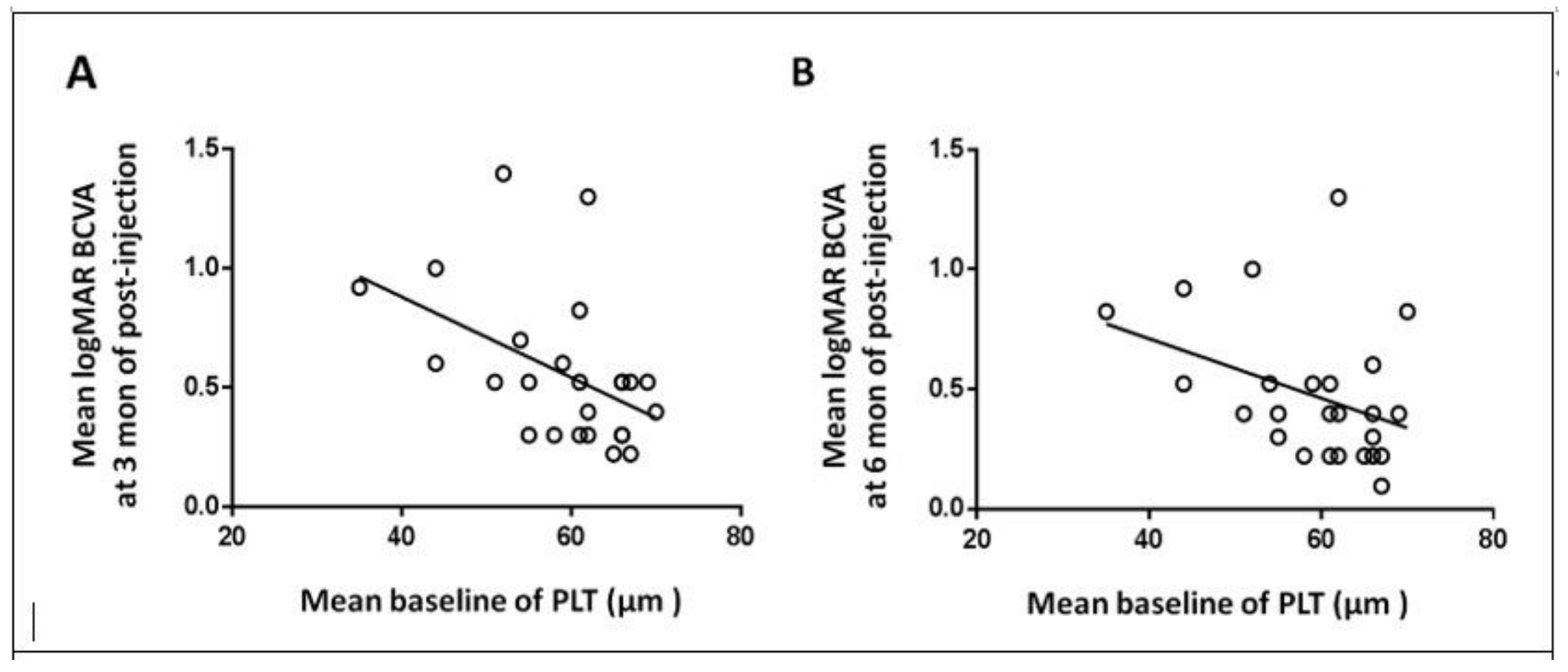

\section{Figure 2}

Association between baseline PLT and logMAR BCVA at month 3 of post-injection $(r=-0.549, P \leq 0.001$, Fig A), Association between baseline PLT and logMAR BCVA at month 3 of post-injection $(r=-0.087, P \leq$ 0.001 , Fig B). 